\title{
Congenital imprinting disorders: EUCID.net - a network to decipher their aetiology and to improve the diagnostic and clinical care
}

\author{
Thomas Eggermann 1,13*, Irène Netchine ${ }^{2,3,4}$, I Karen Temple ${ }^{5}$, Zeynep Tümer ${ }^{6}$, David Monk ${ }^{7}$, Deborah Mackay ${ }^{5}$, \\ Karin Grønskov ${ }^{6}$, Andrea Riccio ${ }^{8,9}$, Agnès Linglart ${ }^{10,11}$ and Eamonn R Maher ${ }^{12}$
}

\begin{abstract}
Imprinting disorders (IDs) are a group of eight rare but probably underdiagnosed congenital diseases affecting growth, development and metabolism. They are caused by similar molecular changes affecting regulation, dosage or the genomic sequence of imprinted genes. Each ID is characterised by specific clinical features, and, as each appeared to be associated with specific imprinting defects, they have been widely regarded as separate entities. However, they share clinical characteristics and can show overlapping molecular alterations. Nevertheless, IDs are usually studied separately despite their common underlying (epi)genetic aetiologies, and their basic pathogenesis and long-term clinical consequences remain largely unknown. Efforts to elucidate the aetiology of IDs are currently fragmented across Europe, and standardisation of diagnostic and clinical management is lacking. The new consortium EUCID.net (European network of congenital imprinting disorders) now aims to promote better clinical care and scientific investigation of imprinting disorders by establishing a concerted multidisciplinary alliance of clinicians, researchers, patients and families. By encompassing all IDs and establishing a wide ranging and collaborative network, EUCID.net brings together a wide variety of expertise and interests to engender new collaborations and initiatives.
\end{abstract}

Keywords: Imprinting disorders, Imprinted genes, Epimutation, Uniparental disomy, EUCID.net, Networking

\section{Review \\ Introduction}

Imprinting disorders (IDs) are a group of eight rare congenital diseases affecting growth, development and metabolism with a lifelong impact on patients' quality of life. They are caused by changes in gene regulation ('epigenetic mutation'), gene dosage and - rarely - in gene or genomic sequences ('genetic mutation') (Figure 1). The term genomic imprinting describes the expression of specific genes in a parent-of-origin-specific manner - that is, they are expressed only from the maternal or from the paternal gene copy, but not biparentally (for review: [1]). The underlying epigenetic basis does not involve the DNA sequence itself, but regulatory mechanisms that ensure

\footnotetext{
* Correspondence: teggermann@ukaachen.de

'Department of Human Genetics, RWTH Aachen, Aachen 52074, Germany

${ }^{13}$ Department of Human Genetics, University Hospital, RWTH Aachen,

Pauwelsstr. 30, 52074 Aachen, Germany

Full list of author information is available at the end of the article
}

the transmission of specific gene expression patterns from one cell generation to another, ensuring the maintenance of cellular identity.

So far, more than 60 human genes have been shown to be imprinted, but there are probably many more (for review: [2]). The normal imprinting marks are inherited from the parental gametes and are then maintained in the somatic cells of an individual. Their programming is subject to an imprinting cycle during life which leads to a reprogramming at each generation (for review: [3]): In early development, methylation of the mammalian genome is comprehensively remodelled, but imprinting marks are exempt from developmental reprogramming; instead, they are erased in the germ line and re-established according to the sex of the contributing parent for the next generation. Many genes regulated by genomic imprinting are found in clusters, that is, imprinted loci often comprise multiple genes under coordinated control. At the molecular level, the expression of genes within imprinted regions 


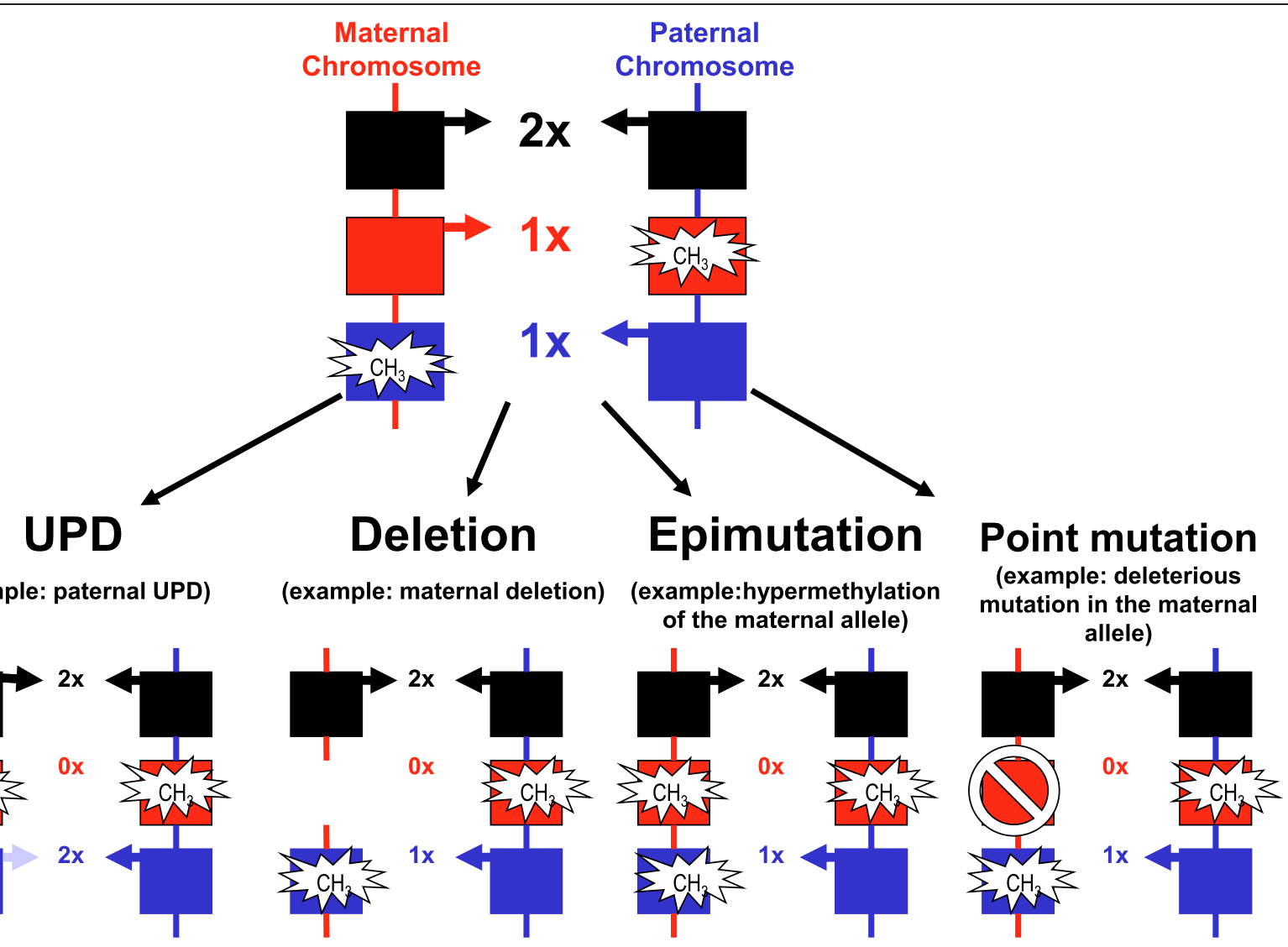

Figure 1 The four molecular mechanisms of IDs, resulting in a disturbed expression of imprinted genes.

is influenced by specific patterns of DNA methylation, by changes in chromatin structure and by post-translational histone modifications, collectively designated as epigenetic regulation (for review: $[4,5]$ ).

The epigenetic machinery is extremely complex and results in a unique transcriptional activity of different cells with identical DNA sequences. Indeed, this carefully orchestrated interplay is prone to various disturbances resulting in distinct pathological courses, for example, malignant tumours or - in the case of parentally imprinted genes - IDs. In IDs, the regulation of imprinted genes can be disturbed by four different molecular alterations: genomic imbalances (duplications/deletions), uniparental disomy (UPD; the inheritance of both homologos of a chromosomal pair from only one parent), epimutations (disturbed methylation) or point mutations in an imprinted gene. Whereas in the majority of ID patients, only the disease-specific loci are affected, an increasing number of ID patients are reported showing a disturbed methylation at multiple differentially methylated regions (DMRs), the so-called multilocus imprinting disturbances (MLID) (see below). The extreme examples of unbalanced imprinting patterns are genome-wide UPDs, that is, the whole genome is inherited only from the father or from the mother.
In both cases, the resulting conception is not viable. However, mosaic genome-wide UPD has been reported to be compatible with life (for review: [6]).

\section{The known imprinting disorders}

Most patients with one of the currently established IDs are diagnosed in early childhood. In contrast, the diagnosis in the prenatal workup or puberty or adulthood is often hampered because the clinical spectrum is broad, and some features are subtle, overlapping and transient. As a result, some IDs are probably mis- and underdiagnosed.

Each ID is characterised by specific clinical features, and as they appeared to be associated with specific imprinting defects, they have been regarded as separate entities. Indeed, the majority of IDs have some shared clinical characteristics (Table 1), that is:

- prenatal and/or postnatal growth retardation or prenatal and postnatal overgrowth;

- hypo- or hyperglycemia;

- abnormal feeding behaviour in early childhood and later; and

- behavioural difficulties in childhood. 
Table 1 Overview on the clinical and molecular characteristics of the currently known eight IDslocalization

\begin{tabular}{|c|c|c|c|c|c|c|c|c|}
\hline $\begin{array}{l}\text { Imprinting } \\
\text { disorder }\end{array}$ & $\begin{array}{l}\text { Alternative } \\
\text { name/acronym }\end{array}$ & Frequency & OMIM & $\begin{array}{l}\text { Chromosomes }{ }^{\mathrm{a}} / \\
\text { imprinted regions }\end{array}$ & Type of mutation/epimutation & MLID & Detection rate & Main clinical features \\
\hline \multirow{3}{*}{$\begin{array}{l}\text { Transient neonatal } \\
\text { diabetes mellitus }\end{array}$} & \multirow[t]{3}{*}{ TNDM } & \multirow[t]{3}{*}{$1 / 300,000$} & \multirow[t]{3}{*}{601410} & \multirow[t]{3}{*}{$6 \mathrm{q} 24^{\mathrm{a}}:$ ZAC1/HYMA1 } & upd(6)pat & & $40 \%$ & \multirow{3}{*}{$\begin{array}{l}\text { IUGR, transient diabetes, } \\
\text { hyperglycemia without ketoacidosis, } \\
\text { macroglossia, omphalocele }\end{array}$} \\
\hline & & & & & Paternal duplications & & $40 \%$ & \\
\hline & & & & & Methylation defects & Approximately 50\% & $20 \%$ & \\
\hline \multirow{5}{*}{$\begin{array}{l}\text { Silver-Russell } \\
\text { syndrome }\end{array}$} & \multirow{5}{*}{$\begin{array}{l}\text { Russell-Silver } \\
\text { syndrome, SRS, RSS }\end{array}$} & \multirow{5}{*}{$\begin{array}{l}1 / 75,000- \\
1 / 100,000\end{array}$} & \multirow[t]{5}{*}{180860} & $7^{\mathrm{a}}$ & upd(7)mat & \multirow[t]{3}{*}{ One case ${ }^{b}$} & Approximately $10 \%$ & \multirow{5}{*}{$\begin{array}{l}\text { IUGR/PNGR, rel. macrocephaly, } \\
\text { hemihypotrophy, triangular face, } \\
\text { feeding difficulties }\end{array}$} \\
\hline & & & & \multirow[t]{2}{*}{$11 p 15^{a}:$} & upd(11p15)mat & & Single cases & \\
\hline & & & & & Maternal duplication & & $<1 \%$ & \\
\hline & & & & IGF2/H19 & Hypomethylation & \multirow[t]{2}{*}{$7 \%$ to $10 \%$} & $>38 \%$ & \\
\hline & & & & CDKNIC & Point mutations & & One family reported & \\
\hline \multirow{5}{*}{$\begin{array}{l}\text { Beckwith-Wiedemann } \\
\text { syndrome }\end{array}$} & \multirow{5}{*}{$\begin{array}{l}\text { Wiedemann-Beckwith } \\
\text { syndrome, EMG } \\
\text { syndrome, BWS }\end{array}$} & \multirow[t]{5}{*}{$1 / 15,000$} & \multirow[t]{5}{*}{130650} & $11 \mathrm{p} 15^{\mathrm{a}}:$ & upd(11p15)pat & & Approximately $20 \%$ & \multirow{5}{*}{$\begin{array}{l}\text { Prenatal and postnatal overgrowth, } \\
\text { organomegaly, macroglossia, } \\
\text { omphalocele, neonatal } \\
\text { hypoglycemia, hemihypertrophy, } \\
\text { increased tumour risk }\end{array}$} \\
\hline & & & & & Chromosomal aberrations & & $2 \%$ to $4 \%$ & \\
\hline & & & & ICR1: IGF2/H19; & Hypermethylation & & $5 \%$ to $10 \%$ & \\
\hline & & & & ICR2:KCNQ1 & Hypomethylation & Approximately $25 \%$ & $40 \%$ to $50 \%$ & \\
\hline & & & & CDKN1C & Point mutations & & $\begin{array}{l}5 \% \text { (sporadic) 40\% } \\
\text { to } 50 \% \text { (families) }\end{array}$ & \\
\hline \multirow{2}{*}{$\begin{array}{l}\text { Kagami-Ogata } \\
\text { syndrome }\end{array}$} & \multirow{2}{*}{$\begin{array}{l}\text { KOS14, upd(14)pat } \\
\text { syndrome }\end{array}$} & \multirow[t]{2}{*}{ Not known } & \multirow[t]{5}{*}{608149} & \multirow[t]{2}{*}{ 14q32 : DLK1/GTL2 } & upd(14)pat & \multirow[t]{2}{*}{ Not yet reported } & \multirow[t]{2}{*}{$?$} & \multirow{2}{*}{$\begin{array}{l}\text { IUGR, polyhydramnion, abdominal } \\
\text { and thoracal wall defects, } \\
\text { bell-shaped thorax, coat-hanger ribs }\end{array}$} \\
\hline & & & & & Aberrant methylation & & & \\
\hline \multirow[t]{3}{*}{ Temple syndrome } & \multirow{3}{*}{$\begin{array}{l}\text { TS14, upd(14)mat } \\
\text { syndrome }\end{array}$} & Not known & & 14q32 & upd(14)mat & One case ${ }^{b}$ & $?$ & IUGR/PNGR, Hypotonia, feeding \\
\hline & & & & & Paternal deletion & & & obesity, scoliosis, precocious \\
\hline & & & & & Aberrant methylation & & & puberty \\
\hline Prader-Willi syndrome & Prader-Labhart-Willi- & $1 / 25,000$ & 176270 & $15 q 11-q 13^{a}$ & Paternal deletion & One case with PWS & $70 \%$ & PNGR, mental retardation, neonatal \\
\hline & & $-1 / 10,000$ & & & upd(15)mat & and BWs features & $<30 \%$ & $\begin{array}{l}\text { hypotonia, hypogenitallsm, } \\
\text { hypopigmentation, obesity/ }\end{array}$ \\
\hline & & & & & Aberrant methylation & & Approximately $1 \%$ & hyperphagia \\
\hline Angelman syndrome & Happy puppet & $1 / 20,000$ & 105830 & $15 q 11-q 13^{a}:$ & Maternal deletion & Not yet reported & $70 \%$ & Mental retardation, microcephaly, \\
\hline & & $-1 / 12,000$ & & & upd(15)pat & & $1 \%$ to $3 \%$ & ataxia, seizures, scoliosis \\
\hline & & & & & Aberrant methylation & & Approximately $4 \%$ & \\
\hline & & & & UBE3A & Point mutations & & $10 \%$ to $15 \%$ & \\
\hline $\begin{array}{l}\text { Pseudohypo- } \\
\text { parathyroidism }\end{array}$ & $\begin{array}{l}\text { PHP1B, PHP1C and } \\
\text { PHP1A }\end{array}$ & Not known & 603233 & $20 \mathrm{q} 13^{\mathrm{a}}$ & Maternally inherited deletions & & Not yet reported & $\begin{array}{l}\text { Resistance to PTH and other } \\
\text { hormones }\end{array}$ \\
\hline & & & 612462 & GNAS & Causing aberrant methylation & & & Albright hereditary osteodystrophy \\
\hline & & & 103580 & & Isolated epimutations & $12.5 \%$ & & Subcutaneous ossifications \\
\hline & & & & & upd(20)pat & & & Feeding behaviour anomalies \\
\hline & & & & & $\begin{array}{l}\text { Maternal and paternal heterozygous } \\
\text { loss of function mutations in the } \\
\text { coding sequence of GNAS }\end{array}$ & & & Abnormal growth patterns \\
\hline
\end{tabular}

As listed in the second column, for several IDs, different names have been proposed. To reach a consensus on a common nomenclature of IDs, EUCID.net has decided to use the disorders names listed on the left (see www.imprinting-disorders.eu). IUGR, intrauterine growth retardation; PNGR, postnatal growth retardation. ${ }^{\mathrm{a} C h r o m o s o m e s}$. ${ }^{\mathrm{b}}$ Case [27] carries both upd(7)mat and an TS14 epimutations. 


\section{Transient neonatal diabetes mellitus}

Transient neonatal diabetes mellitus (TNDM) is a rare disease, characterised as its name implies by transient hyperglycaemia. In addition, IUGR, macroglossia and abdominal wall defects are common. Insulin therapy is required for an average of 3 months; afterwards, the diabetes resolves, but later in life, the majority of TNDM patients develop type 2 diabetes. TNDM is associated with an overexpression of PLAGL1/ZAC in 6q24, a maternally imprinted gene. It encodes a zinc-finger protein which binds DNA and hence influences the expression of other genes (for review: $[7,8]$ ).

\section{Silver-Russell syndrome}

Silver-Russell syndrome (SRS) is mainly characterised by prenatal and postnatal growth restriction with relative macrocephaly at birth, severe feeding difficulties during early childhood and typical facial gestalt and, in many cases, asymmetry. The genetic basis of SRS is heterogeneous. In approximately $10 \%$ of SRS patients, a maternal UPD for chromosome 7 [upd(7)mat] can be found (for review: $[9,10])$. More than $40 \%$ of SRS patients show a hypomethylation of the ICR1 DMR in the imprinted region 11p15. In single cases, genomic alterations in 11p15 chromosomal region have been reported (for example, maternal duplications). Additionally, numerous (submicroscopic) disturbances of other chromosomes than 7 and 11 have been described in SRS patients; thus, screening for cryptic genomic imbalances is indicated after exclusion of upd(7)mat and 11p15 epimutations $[11,12]$. Furthermore, there is an overlap with Temple syndrome (upd(14)mat). The genes causing the SRS phenotype on chromosomes 7 and 11 are currently unknown, but a role of IGF2 and CDKN1C in $11 \mathrm{p} 15$ has been suggested.

\section{Beckwith-Wiedemann syndrome}

Beckwith-Wiedemann syndrome (BWS) was initially called EMG syndrome from its three main features of exomphalos, macroglossia and (neonatal) gigantism. In $5 \%$ to $7 \%$ of children, embryonal tumours (most commonly Wilms tumour) are diagnosed. The clinical diagnosis of BWS is often difficult due to its variable presentation and the phenotypic overlap with other overgrowth syndromes (for review: $[13,14]$ ). In nearly $70 \%$ of BWS patients, an altered expression or mutations of several loci in $11 \mathrm{p} 15$ can be observed (including the ICR1 and ICR2 DMRs). ICR2 hypomethylation account for about $50 \%$ of the cases, upd(11p15)pat is the second most frequent molecular aberration, while ICR1 hypermethylation is less frequent ( $2 \%$ to $7 \%)$. Most BWS cases are sporadic, but familial inheritance is observed in 15\% of all cases. Microdeletions/duplications or point mutations at the ICRs are usually found in familial BWS with aberrant 11p15 methylation, while CDKN1C mutations are frequent in familial cases with normal 11p15 methylation $[15,16]$. These BWS pedigrees resemble that of an autosomal dominant inheritance but with incomplete penetrance depending on the sex of the inheriting parent. A genotype/epigenotype-phenotype correlation has recently been established for BWS [17]: hemihypertrophy is strongly associated with upd(11)pat, exomphalos with ICR2 hypomethylation and CDKN1C mutations, and, most importantly, the risk of Wilms tumour is significantly higher in ICR1 hypermethylation and upd(11)pat than in the other molecular subgroups. In BWS, the determination of the molecular subtype is therefore important for an individual prognosis and therapy. Nevertheless, the phenotypic transitions are fluid, and testing for all molecular subtypes should be offered in patients with BWS features.

\section{Temple syndrome (TS14) [upd(14)mat]) and Kagami-Ogata syndrome (KOS14) [upd(14)pat]}

TS14 (upd(14)mat and WGS (upd(14)pat) were described in 1991 by Temple et al. and Wang et al. $[18,19]$, respectively. However, the frequencies of both syndromes are currently unknown. Both IDs were first detected in patients carrying balanced Robertsonian translocations. Considering the most important formation mechanism of UPD via trisomy rescue, this observation was consequent because Robertsonian translocations are prone to trisomic offspring. More recently, several cases have been described with microdeletions affecting $14 \mathrm{q} 32$ or with isolated methylation anomalies affecting the imprinting control region $[20,21]$.

Among other clinical signs, TS14 is characterised by prenatal and postnatal growth retardation, muscular hypotonia, feeding difficulties in early childhood, truncal obesity and early onset of puberty. TS14 patients show clinical features overlapping with PWS and SRS, and thus, screening for chromosome $14 \mathrm{q} 32$ should be performed in patients with PWS- and SRS-like phenotypes after exclusion of the specific (epi)mutations.

KOS14 is associated with polyhydramnios, a characteristic small, bell-shaped thorax, abdominal wall defects and a severe developmental delay. Many patients have been reported to die in utero or in the first months of life, but exceptions exist.

For both syndromes, the role of an altered RTL1 and DLK1 expression has been suggested [21].

\section{Angelman and Prader-Willi syndromes}

Both Angelman syndrome (AS) and Prader-Willi syndrome (PWS) are caused by (epi)mutations in 15q11-q13. The lack of the paternal contribution of this region results in PWS, while lack of the maternal contribution leads to AS. Both AS and PWS patients are mentally retarded, but the 
remaining clinical signs are different. PWS is clinically characterised by neonatal hypotonia and failure to thrive in infancy, with subsequent development of hyperphagia and obesity (for review: [22]). Approximately $70 \%$ of individuals with PWS have an interstitial deletion of the paternal 15q11-q13 allele, $20 \%$ to $30 \%$ have maternal UPD of 15 q11-q13 while $<1 \%$ have an imprinting defect (either primary or secondary). As mentioned before, analysis for TS14 should be considered in PWS patients without chromosome 15 disturbances.

AS patients exhibit microcephaly, ataxia, seizures, absence of speech and sleep disorder (for review: [23]). Deletion of the maternal copy of 15q11-q13 is observed in approximately $70 \%$ of individuals with AS, paternal UPD in $7 \%$ to $10 \%$, while $3 \%$ have an imprinting defect (either primary or secondary). Around $10 \%$ have mutations in $U B E 3 A$.

Due to the high percentage of microdeletions in 15q11q13 in both syndromes, AS and PWS also belong to the so-called microdeletion syndromes, a group of congenital disorders caused by a chromosomal deletion spanning several genes but too small to be detected by conventional cytogenetic.

\section{Pseudohypoparathyroidism}

Pseudohypoparathyroidism (PHP) is a group of disorders united by parathyroid hormone (PTH) resistance in the kidney, that is, pseudohypoparathyroidism. Most cases of PHP belong to the type 1, that is, are caused by genetic or epigenetic alterations at the imprinted GNAS locus. PHP1A comprises patients affected with resistance to PTH and thyroid stimulating hormone (TSH) and features of obesity and Albright's hereditary osteodystrophy including short stature, brachydactyly, ectopic ossifications and mental retardation. PHP1A is due to loss of function mutations in the maternal allele of the GNAS gene. Paternal GNAS mutations are associated with $\mathrm{AHO}$, no hormonal resistance and no obesity. In contrast, the phenotype of most PHP1B patients is limited to renal PTH resistance and in some cases, mild TSH resistance. Few patients with PHP1B display some features of Albright's hereditary osteodystrophy. Patients with PHP1B share a loss of methylation at the A/B DMR of GNAS, likely leading to the downregulated expression of the GNAS-Gsa transcript in imprinted tissues. Some patients carry additional epigenomic changes along the GNAS locus. About 20\% of PHP1B are inherited and due to deletions of GNAS imprinting control regions. The remaining $80 \%$ are sporadic. A small subset is due to paternal UPD of chromosome 20q, yet the vast majority are still of unknown cause. While obesity and short stature are long known features of PHP1A, it became only recently apparent that growth and metabolism are affected in both paternal and maternal epi/genetic alterations of the GNAS locus (for review: $[24,25]$ ).

\section{Molecular alterations in IDs}

In nearly all known IDs, the same classes of molecular changes are detectable. Broadly, the several mechanisms have an underlying genetic lesion, but a considerable number have no identifiable genetic cause, and are reproductive, stochastic or epigenetic in origin (Figure 1).

The genetic lesions include:

a) chromosomal deletions, duplications and rearrangements;

b) intragenic mutations in imprinted genes.

In familial cases of these genetically caused IDs, parent of origin dependence of expression results in apparent nonMendelian inheritance.

The non-genetic causes consist of:

c) UPD (that is, the inheritance of both chromosomal homologos from the same parent);

d) epimutations (that is, aberrant methylation without alteration of the genomic DNA sequence).

It is noteworthy that non-genetic aberrations may occur post-zygotically, resulting in a mosaic distribution. Mosaicism can obscure genotype-phenotype correlation, and is also associated with somatic asymmetry, and discordant monozygotic twinning.

For genetic counselling of ID families, the knowledge of the nature of the mutation or epimutation subtype is essential to delineate exact risk figures. Whereas the recurrence risk is generally low in the case of epimutations and UPD, patients/carriers with submicroscopic deletions or duplications might have a $50 \%$ risk of conceiving a child with an ID, depending on the sex of the contributing patient. However, in each case, genetic professionals are advised to continually update their knowledge for each disease.

Each of the currently known IDs was initially reported to be associated with molecular alterations at specific chromosomal loci. They have therefore been regarded as separate entities, but - as mentioned before - with the growing data on IDs, it becomes apparent that they share both genetic properties and clinical features. This can cause uncertainty determining which molecular tests to perform and with what priority, particularly for patients with growth restriction. Moreover, the clinical overlap between the different IDs is reflected on molecular level by the identification of similar multilocus methylation defects in different phenotypes. In particular, in growth retarded patients, it is sometimes difficult to decide which ID-specific test should be applied.

Multilocus imprinting disturbances, a common finding in IDs The correlation between aberrations at specific imprinted genes and distinct congenital disorders was generally accepted for nearly 20 years, but there are growing numbers 
of reports on patients with generally disturbed imprinting patterns (MLID) (Table 1) (for review: [26]). These patients often exhibit a specific ID phenotype, for example, BWS, but molecular testing reveals that aberrant methylation does not affect only the disease-specific imprinted loci (for example, 11p15 in BWS) but also other imprinted regions. Remarkably, patients with opposite phenotypes like BWS (overgrowth) and SRS (growth retardation) can share some aberrant methylation patterns in lymphocytes (lymphocyte DNA is the most frequently and often the only analysed tissue), and to add a layer of complexity, epigenotype anomalies can vary for the same individual depending on the studied tissue. This has been so far investigated in SRS patients with 11p15 ICR1 LOM identified initially in leukocytes. Another example is the phenotype of an upd (7q)mat carrier with hypomethylation in $14 \mathrm{q} 32$ who was initially diagnosed as SRS (typical for upd(7q)mat but then exhibited a phenotype suggestive for TS14 [27].

In summary, the clinical picture in patients with methylation aberrations affecting more than one imprinted locus can be different from that of patients with single epimutations, but is not necessarily so. As a result, both patients with typical ID phenotypes as well as those with unusual clinical features should be tested for MLID.

Two genetic mechanisms causing aberrant methylation at imprinted loci have been identified: cis- and trans-acting alterations. Cis-acting alterations affect DNA sequences which are physically localised adjacent to a structural gene or its control regions and interact with them. Examples for cis-acting mutations are rare deletions of CTCF-binding sites and mutations affecting the OCT- and SOX-binding elements in 11p15 which mediate the regulation of ICR1: such mutations affect up to $20 \%$ of BWS patients with 11p15 ICR1 GOM [28], but the BWS phenotype is expressed only if the mutation affects the maternal chromosome 11 $[29,30]$. Trans-acting factors also influence the expression of genes, but they act by intermediary diffusible molecules (proteins, RNAs). Their genes can be localised on the same chromosome as the target gene or elsewhere in the genome. Several trans-acting factors have been postulated to cause MLID (for review: [26]), and indeed, DNA mutations in factors involved in the imprinting cycle have been reported [31-33].

In addition to these monogenic causes of aberrant imprinting, the identification of patients with MLID corroborates the hypothesis of an 'imprinted gene network', that is, a network of interacting imprinted genes and regions [34]. By this network, the disturbance of one member alters the regular expression of the others.

\section{The COST Action BM1208: EUCID.net - a network of European groups working in the field of IDs}

Despite their common underlying (epi)genetic aetiologies, IDs are usually studied separately by small groups working in isolation, and the basic pathogenesis and long-term clinical consequences of IDs remain largely unknown. Efforts to elucidate the aetiology of IDs are currently fragmented across Europe, and standardisation of diagnostic and clinical management is lacking.

To overcome this fragmentation and to achieve a consensus in diagnostic and treatment of IDs, European groups working on IDs and epigenetic regulation have established a network, called EUCID.net (European network for human congenital imprinting disorders; www.imprinting-disorders. eu), which, for the first time, draws together researchers of all eight known human IDs in an interdisciplinary activity, working to advance understanding of the pathophysiology with the major aim of translating this knowledge to improvement of diagnostic and clinical management for the benefit of the patients and their families. The Action will harmonise a common system for clinical and molecular classification as well as nomenclature of IDs, develop guidelines for treatment through consensus, create standard operating procedures (SOPs) for diagnosis based on best current practice, coordinate databases held in different countries to make them compatible and useful as a springboard for collective research initiatives, identify new imprinting disorders through collaborative effort, educate researchers and stimulate translational exchange. These networking activities have become possible with funding of COST, the European Cooperation in Science and Technology, (COST Action BM1208).

\section{Objectives of EUCID.net}

The objectives of the network will be realised in five working groups (WGs) described below. The activities will run in three interdependent directions: (a) Clinical experts are undertaking the challenging task of standardisation and harmonisation of clinical phenotyping and medical management of IDs, providing guidelines for IDs' clinical assessment and management across Europe. (b) Experts in molecular diagnosis are undertaking the standardisation of molecular diagnosis of IDs and development of consistent reproducible molecular testing. (c) A coordinated European infrastructure of data sharing (clinical and molecular data) and samples for genetic and epigenetic study of IDs will be created (with the possibility to link clinical, genetic and epigenetic data). This will be an important step towards improving the standard of care for IDs in Europe and uncovering the genetic/epigenetic bases of the disorders.

\section{WG1 - European clinical integration}

By European wide cooperation and coordination, this WG will gather Europe-wide experience in the clinical and metabolic characteristics and management practices of ID patients to provide a comprehensive clinical review of IDs. This will enable the development of standardised 
recording of phenotypes across centres and the development of a disease classification enabling future improvements in diagnosis. We will also strive for a current consensus on clinical management guidelines on which to build as new developments emerge. With the publication of disease-specific clinical utility cards, the first step has been undertaken [35-40].

\section{WG2 - molecular biology}

This multidisciplinary WG will unify recording of samples to create a virtual biobank for IDs across Europe, creating pan-European resources for the study of the (epi)genetic basis of IDs. The heterogeneity and complexity of IDs demands the availability of large patient cohorts which has not been available until now. Furthermore, this WG will attempt to unify the types of the biological material obtained from affected families (DNA, RNA, transformation of lymphocytes). This will allow the identification of factors commonly involved in the aetiology of IDs. This WG will focus on activities aiming on the identification of new genes, cis-acting control elements and epigenetic trans-acting factors associated with and/or causing IDs as a prerequisite for an understanding of pathophysiological mechanisms. Unravelling the cross talk between known and new ID genes can place these disorders in an (epi)genomic perspective. This WG will coordinate genomic, epigenomic, proteomic and transcriptomic studies by high-throughput assays. By data exchange, these new findings and techniques will be implemented in clinical and diagnostic application.

\section{WG3 - molecular diagnostics}

WG3 will discuss important technical aspects related to molecular diagnosis of IDs, aiming to provide a harmonised testing algorithm for IDs. The partners in WG3 will implement innovative diagnostic algorithms by improvement of existing ID-specific tests and by development of new tests for specific IDs. The diagnostic utility of these techniques will be validated, and standardised algorithms will be progressively incorporated into diagnostic regimes through collective experience. Reference panels and quality control measures will be established in cooperation with the European Molecular Quality Network (EMQN). It is expected that most European experts in the field of IDs are going to participate in this effort and they will collaborate with experts around the world, thus turning this COST Action into a global initiative.

\section{WG4 - capacity building}

Being the springboard for new research ideas, this WG will use the combined experiences of COST members to initiate new directions and projects in ID research responding to research calls through the preparation and submission of grant proposals to European and international funding agencies. Furthermore, networking activities and short-term scientific missions will be organised to strengthen the interdisciplinary and transnational activities.

\section{WG5 - dissemination}

This WG will design and coordinate outreach meetings with patient groups and dissemination activities. It will receive input from the other WGs and channel their scientific efforts into public dissemination. The publication of new disease classification systems and clinical guidelines, together with coordinated diagnostics, will represent a major objective for delivering individualized therapeutic management. This WG will promulgate the results, guidelines and clinical classifications to physicians, scientists and patients' organisations. WG5 will have close links with national patients' organisations.

\section{Organisation}

At the beginning in May 2013, 25 groups from 11 European countries have been part of EUCID.net, including academic groups, SMEs and patients' organisations. Until July 2014, additional groups have joined the network, and it currently includes 45 groups from 22 European countries. Furthermore, there are close links and exchanges with experts from Australia, Canada, Japan, South America and the USA.

Based on the COST rules, the EUCID.net includes the following partcipants (Figure 2): A Chair and a ViceChair, both elected, who preside over the management committee (MC). The $M C$ coordinates the key issues of the Action. It consists of two representatives per participating country, including the Coordinator and Co-Coordinator of each WG. It is responsible for the allocation of funds and oversees for the overall strategy of the network. The MC manages operations of the WGs, the programme of international symposia, as well as training and exchange programmes. The $\mathrm{MC}$ fosters the exchange of scientific knowledge and active collaborators. Due to its smaller size, the steering committee (SC), consisting of the WG co/ coordinators, represents a more flexible instrument that allows the close monitoring of the progress of the Action and acts as a link between the WGs and the different groups. Under the directions of the MC, the SC is furthermore responsible for the interaction with existing platforms in Europe and across the world and relevant stakeholders (for example, IRDiRC, EURORDIS, Orphanet, EMQN, EUCERD). Each of the five WGs is chaired by a WG leader (Coordinator) and a co-leader who have been elected by the $\mathrm{MC}$ during the kick-off meeting. Leader and co-leader are responsible for the coordination, organisation and supervision of the WG's meetings. Each WG is constituted by different teams, but the teams can be involved in different WGs. All members of each WG will meet once a year 


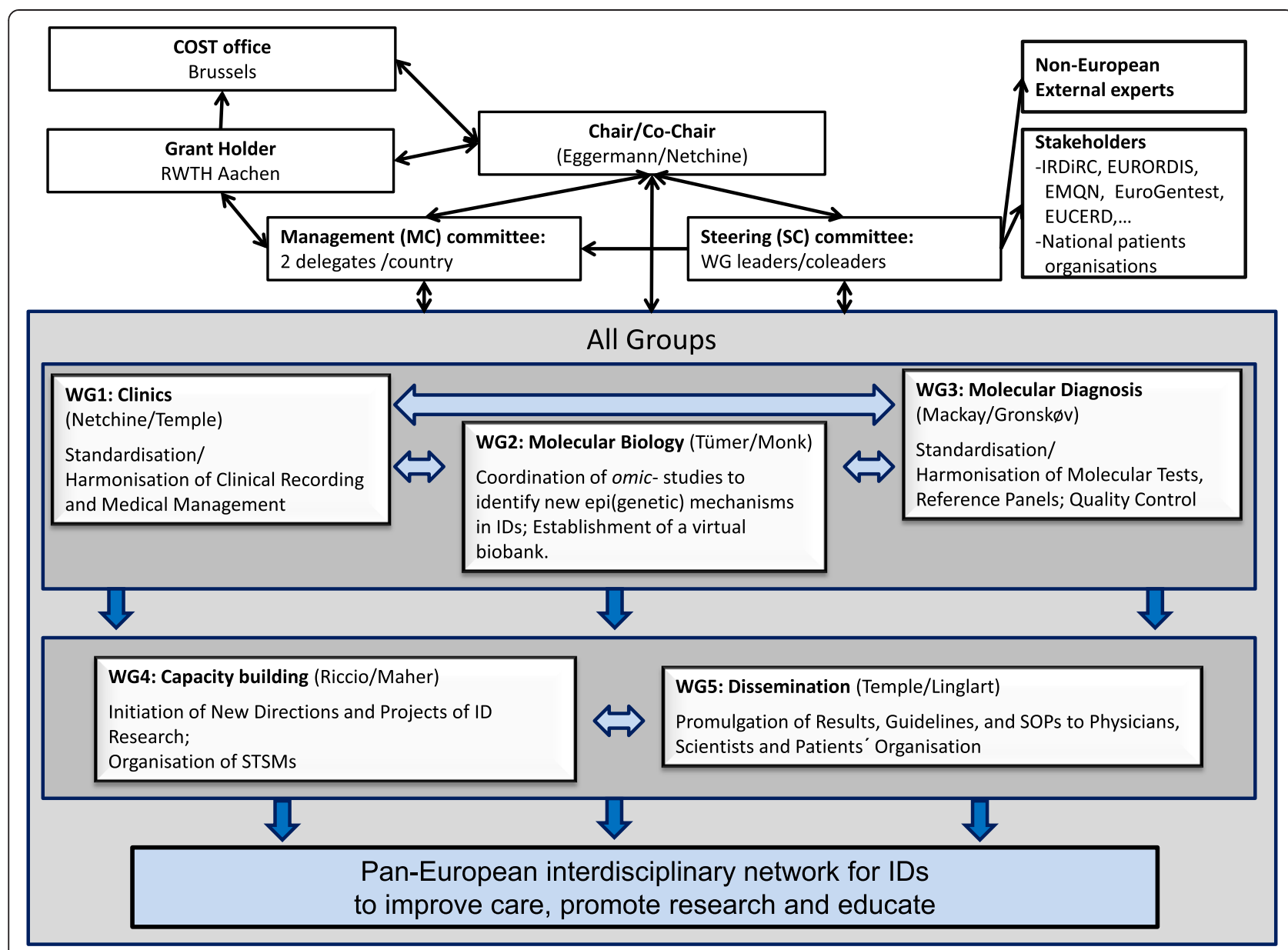

Figure 2 Structure and activities of the EUCID.net/COST BM1208, all aiming on networking of researchers, clinicians, SMEs and patients organisation working in the field of IDs.

to establish the guidelines and SOPs as well as to exchange scientific knowledge and data. The investigators of each WG communicate through regular conference calls and web-based interfaces. All five WGs are interrelated and interact through the SC. WG 1 is closely related to WG 2 and 3, establishing tools and guidelines for phenotype characterization. In turn, WG 2 and 3 are connected to deciphering the molecular basis of IDs and to translate the achieved knowledge into diagnostic application. Furthermore, they contribute to the ID classification and development of clinical guidelines in WG 1. All efforts from WG1 3 support and stimulate the activities in WG 4 to initiate new directions and projects. WG1 4 support WG 5 in the promulgation of the data and achieved knowledge, inter alia by implementation of WG specific information on the website in a password protected area.

High priority is given to Early Stage Researchers (ESRS) and Short-Term Scientific Missions (STSMs) for maximizing the exchange of experience among the participants as exchanging ideas and knowledge across borders will lead to more successful projects. The ID training school is a further central instrument to pursue these aims.

\section{Conclusions}

Imprinting disorders are underdiagnosed, and currently available diagnostic and management protocols are suboptimal. Improvements in the diagnosis and management of rare diseases are greatly facilitated by international collaboration. EUCID.net aims to promote better clinical care and scientific investigation of imprinting disorders by establishing a concerted multidisciplinary alliance of clinicians, researchers, patients and families. By encompassing all IDs and establishing a wide ranging, open and collaborative network, EUCID will bring together a wide variety of expertise and interests to engender new collaborations and initiatives. It is very much hoped that epigeneticists with an interest in imprinting disorders will wish to participate in EUCID.net (contact: teggermann@ukaachen.de). 


\section{Competing interests}

The authors declare that they have no competing interests.

\section{Authors' contributions}

TE and ERM prepared the draft. All authors approved the content and contributed their expertise in the different fields of imprinting disorders, that is, TE, DM and IN in the field of Silver-Russell syndrome, ZET and KG for the chromosome 15 disorders, AR, IN and ERM for the Beckwith-Wiedemann syndrome, IKT and DJM for the chromosome 14 syndromes and TNDM, and AL for the PHP. All authors read and approved the final manuscript.

\section{Acknowledgements}

All authors are members of the EUCID.net network, funded by the COST (BM1208).

\section{Author details}

'Department of Human Genetics, RWTH Aachen, Aachen 52074, Germany. ${ }^{2}$ INSERM, UMR_S 938, CDR Saint-Antoine, Paris F-75012, France. ${ }^{3}$ UMR_S 938, CDR Saint-Antoine, UPMC Univ Paris 06, Sorbonne Universites, Paris F-75012, France. ${ }^{4}$ Pediatric Endocrinology, 3APHP, Armand Trousseau Hospital, Paris 75012, France. ${ }^{5}$ Human Genetics and Genomic Medicine, Faculty of Medicine University of Southampton, Wessex Clinical Genetics Service, Princess Anne Hospital, Coxford Road, Southampton SO16 5YA, UK. ${ }^{6}$ Clinical Genetic Clinic, Kennedy Center, Rigshospitalet, Copenhagen University Hospital, Glostrup 2600, Denmark. ${ }^{7}$ Imprinting and Cancer Group, Cancer Epigenetic and Biology Program (PEBC), Institut d'Investigació Biomedica de Bellvitge (IDIBELL), Hospital Duran i Reynals, 08907 Barcelona, Spain. ${ }^{8}$ DiSTABiF, Seconda Università degli Studi di Napoli, 81100 Caserta, Italy. ${ }^{9}$ Institute of Genetics and Biophysics-ABT, CNR, Napoli, Italy. ${ }^{10}$ Endocrinology and Diabetology for Children and Reference Center for Rare Disorders of Calcium and Phosphorus Metabolism, Bicêtre Paris Sud, APHP, Le Kremlin-Bicêtre 94276Paris, , France. ${ }^{11}$ INSERM U986, INSERM, Le Kremlin-Bicêtre, 94276 Paris, France. ${ }^{12}$ Department of Medical Genetics, NIHR Cambridge Biomedical Research Centre, University of Cambridge, Cambridge CB2 OXY, UK. ${ }^{13}$ Department of Human Genetics, University Hospital, RWTH Aachen, Pauwelsstr. 30, 52074 Aachen, Germany.

\section{Received: 12 September 2014 Accepted: 26 January 2015} Published online: 14 March 2015

\section{References}

1. Reik W, Walter J. Genomic imprinting: parental influence on the genome. Nat Rev Genet. 2001;2:21-32.

2. Horsthemke B. Mechanisms of imprint dysregulation. Am J Med Genet C. 2010;154C:321-8.

3. Demars J, Gicquel C. Epigenetic and genetic disturbance of the imprinted 11 15 region in Beckwith-Wiedemann and Silver-Russell syndromes. Clin Genet. 2012;81:350-61.

4. Delaval K, Wagschal A, Feil R. Epigenetic deregulation of imprinting in congenital diseases of aberrant growth. Bioessays. 2006;28:453-9.

5. Kacem S, Feil R. Chromatin mechanisms in genomic imprinting. Mamm Genome. 2009;20:544-56.

6. Kalish JM, Conlin LK, Bhatti TR, Dubbs HA, Harris MC, Izumi K, et al. Clinical features of three girls with mosaic genome-wide paternal uniparental isodisomy. Am J Med Genet A. 2013;161:1929-39.

7. Temple IK, Gardner RJ, Robinson DO, Kibirige MS, Ferguson AW, Baum JD, et al. Further evidence for an imprinted gene for neonatal diabetes localised to chromosome 6q22-q23. Hum Mol Genet. 1996;5:1117-21.

8. Temple IK, Shield JP. 6 q24 transient neonatal diabetes. Rev Endocr Metab Disord. 2010;11:199-204.

9. Netchine I, Rossignol S, Dufourg MN, Azzi S, Rousseau A, Perin L, et al. 11 p15 imprinting center region 1 loss of methylation is a common and specific cause of typical Russell-Silver syndrome: clinical scoring system and epigenetic-phenotypic correlations. J Clin Endocrinol Metab. 2007;92:3148-54.

10. Abu-Amero S, Monk D, Frost J, Preece M, Stanier P, Moore GE. The genetic aetiology of Silver-Russell syndrome. J Med Genet. 2008;45:193-9.

11. Bruce S, Hannula-Jouppi K, Puoskari M, Fransson I, Simola KO, Lipsanen-Nyman M, et al. Submicroscopic genomic alterations in Silver-Russell syndrome and Silver-Russell-like patients. J Med Genet. 2010;47:816-22.
12. Spengler S, Begemann M, Ortiz Brüchle N, Baudis M, Denecke B, Kroisel PM, et al. Molecular karyotyping as a relevant diagnostic tool in children with growth retardation with Silver-Russell features. J Pediatr. 2012;161:933-42.

13. Cooper WN, Luharia A, Evans GA, Raza H, Haire AC, Grundy R, et al. Molecular subtypes and phenotypic expression of Beckwith-Wiedemann syndrome. Eur J Hum Genet. 2005;13:1025-32.

14. Choufani S, Shuman C, Weksberg R. Beckwith-Wiedemann syndrome. Am J Med Genet. 2010;154C:343-54.

15. Sparago A, Cerrato F, Vernucci M, Ferrero GB, Silengo MC, Riccio A. Microdeletions in the human H19 DMR result in loss of IGF2 imprinting and Beckwith-Wiedemann syndrome. Nat Genet. 2004;36:958-60.

16. Demars J, Shmela ME, Rossignol S, Okabe J, Netchine I, Azzi S, et al. Analysis of the IGF2/H19 imprinting control region uncovers new genetic defects, including mutations of OCT-binding sequences, in patients with 11 p15 fetal growth disorders. Hum Mol Genet. 2010;19:803-14.

17. Ibrahim A, Kirby G, Hardy C, Dias RP, Tee L, Lim D, et al. Methylation analysis and diagnostics of Beckwith-Wiedemann syndrome in 1,000 subjects. Clin Epigenetics. 2014;6:11.

18. Temple IK, Cockwell A, Hassold T, Pettay D, Jacobs P. Maternal uniparental disomy for chromosome 14. J Med Genet. 1991;28:511-4.

19. Wang JC, Passage MB, Yen PH, Shapiro LJ, Mohandas TK. Uniparental heterodisomy for chromosome 14 in a phenotypically abnormal familial balanced 13/14 Robertsonian translocation carrier. Am J Hum Genet. 1991;48:1069-74

20. Temple IK, Shrubb V, Lever M, Bullman H, Mackay DJ. Isolated imprinting mutation of the DLK1/GTL2 locus associated with a clinical presentation of maternal uniparental disomy of chromosome 14. J Med Genet. 2007;44:637-40.

21. Kagami M, Sekita Y, Nishimura G, Irie M, Kato F, Okada M, et al. Deletions and epimutations affecting the human $14 q 32.2$ imprinted region in individuals with paternal and maternal upd(14)-like phenotypes. Nat Genet. 2008;40:237-42.

22. Goldstone AP. Prader-Willi syndrome: advances in genetics, pathophysiology and treatment. Trends Endocrinol Metab. 2004;15:12-20.

23. Williams CA, Beaudet AL, Clayton-Smith J, Knoll JH, Kyllerman M, Laan LA, et al. Angelman 2005: updated consensus for diagnostic criteria. Am J Med Genet. 2005;2006(140A):413-8.

24. Kelsey G. Imprinting on chromosome 20: tissue-specific imprinting and imprinting mutations in the GNAS locus. Am J Med Genet. 2010;154C:377-86.

25. Linglart A, Maupetit-Méhouas S, Silve C. GNAS-related loss-of-function disorders and the role of imprinting. Horm Res Paediatr. 2013;29:119-29.

26. Eggermann T, Heilsberg AK, Bens S, Siebert R, Beygo J, Buiting K, et al. Additional molecular findings in 11p15-associated imprinting disorders: an urgent need for multi-locus testing. J Mol Med. 2014;92:769-77.

27. Begemann M, Spengler S, Kordaß U, Schröder C, Eggermann T. Segmental maternal uniparental disomy 7q associated with DLK1/GTL2 (14q32) hypomethylation. Am J Med Genet A. 2012;158A:423-8.

28. Abi Habib W, Azzi S, Brioude F, Steunou V, Thibaud N, Neves CD, et al. Extensive investigation of the IGF2/H19 imprinting control region reveals novel OCT4/SOX2 binding site defects associated with specific methylation patterns in Beckwith-Wiedemann syndrome. Hum Mol Genet. 2014;23:5763-73.

29. Beygo J, Citro V, Sparago A, De Crescenzo A, Cerrato F, Heitmann M, et al. The molecular function and clinical phenotype of partial deletions of the IGF2/H19 imprinting control region depends on the spatial arrangement of the remaining CTCF-binding sites. Hum Mol Genet. 2013;22:544-57.

30. Poole RL, Leith DJ, Docherty LE, Shmela ME, Gicquel C, Splitt M, et al. Beckwith-Wiedemann syndrome caused by maternally inherited mutation of an OCT-binding motif in the IGF2/H19-imprinting control region, ICR1. Eur J Hum Genet. 2012;20:240-3.

31. Meyer E, Lim D, Pasha S, Tee LJ, Rahman F, Yates JR, et al. Germline mutation in NLRP2 (NALP2) in a familial imprinting disorder (Beckwith-Wiedemann Syndrome). PLoS Genet. 2009;5:e1000423.

32. Mackay DJ, Callaway JL, Marks SM, White HE, Acerini CL, Boonen SE, et al. Hypomethylation of multiple imprinted loci in individuals with transient neonatal diabetes is associated with mutations in ZFP57. Nat Genet. 2008;40:949-51.

33. Murdoch S, Mazhar B, Seoud M, Khan R, Kuick R, Bagga R, et al. Mutations in NALP7 cause recurrent hydatidiform moles and reproductive wastage in humans. Nat Genet. 2006;38:300-2.

34. Varrault A, Gueydan C, Bellmann A, Houssami S, Aknin C, Severac D, et al. Zac1 regulates an imprinted gene network critically involved in the control of embryonic growth. Dev Cell. 2006;11:711-22. 
35. Eggermann T, Buiting K, Temple IK. Clinical utility gene card for: Silver-Russell syndrome. Eur J Hum Genet. 2011;19(3).

36. Eggermann T, Algar E, Lapunzina P, Mackay D, Maher ER, Mannens M, et al. Clinical utility gene card for: Beckwith-Wiedemann syndrome. Eur J Hum Genet. 2014;22(3).

37. Mackay D, Bens S, Perez de Nanclares G, Siebert R, Temple IK Clinical utility gene card for: transient Neonatal Diabetes Mellitus, 6q24-related. Eur J Hum Genet. 2014;26. doi:10.1038/ejhg.2014.27.

38. Buiting K, Cassidy SB, Driscoll DJ, Gillessen-Kaesbach G, Kanber D, Tauber M, et al. Clinical utility card for: Prader-Willi syndrome. Eur J Hum Genet. 2014; 16. doi:10.1038/ejhg.2014.66.

39. Buiting K, Clayton-Smith J, Driscoll DJ, Gillessen-Kaesbach G, Kanber D, Schwinger E, et al. Clinical utility gene card for: Angelman syndrome. Eur J Hum Genet. 2014;4. doi:10.1038/ejhg.2014.93.

40. Mantovani G, Linglart A, Garin I, Silve C, Elli FM, de Nanclares GP. Clinical utility gene card for: pseudohypoparathyroidism. Eur J Hum Genet. 2013 Jun;21(6). doi:10.1038/ejhg.2012.211. Epub 2012 Sep 12.

\section{Submit your next manuscript to BioMed Central and take full advantage of:}

- Convenient online submission

- Thorough peer review

- No space constraints or color figure charges

- Immediate publication on acceptance

- Inclusion in PubMed, CAS, Scopus and Google Scholar

- Research which is freely available for redistribution 